\title{
Use of Nanocomposite Material Based on Graphene Oxide and Silver Nanoparticles in Research of Blood Erythrocytes in Various Diseases
}

\section{S.N. Mamaeva ${ }^{1}$, G.V. Maksimov², E.P. Neustroev¹, Y.A. Munkhalova¹, S.R.Antonov ${ }^{1}$, and A.N. Pavlov ${ }^{1}$}

${ }^{1}$ North-Eastern Federal University (NEFU), 58 Belinsky street, Yakutsk, Republic of Sakha (Yakutia), Russia

${ }^{2}$ Lomonosov Moscow State University, GSP-1, Leninskie Gory, Moscow, Russia

\section{Abstract}

The electrical and structural properties of a nanocomposite material based on silver nanoparticles and graphene oxide were investigated. Using electron and atomic force microscopy it was shown that the nanoparticles formed predominantly have sizes

Corresponding Author:

S.N. Mamaeva

sargylana_mamaeva@mail.ru

Received: 17 January 2018

Accepted: 25 March 2018

Published: 17 April 2018

Publishing services provided by Knowledge $\mathrm{E}$

(c) S.N. Mamaeva et al. This article is distributed under the terms of the Creative Commons Attribution License, which permits unrestricted use and redistribution provided that the original author and source are credited.

Selection and Peer-review under the responsibility of the PhysBioSymp17 Conference Committee.
G OPEN ACCESS from 60 to $100 \mathrm{~nm}$. Measurements of the current-voltage characteristics showed electrical conductivity values significantly exceeding the parameters of the initial graphene oxide. The prepared nanocomposites were used for scanning by electron microscopy (SEM) of blood samples of sick children with hematuria syndrome and patients of the radiological department of an oncologic dispensary diagnosed with cervical cancer. The formation of nanosize objects on the surface of erythrocytes is revealed. The size of these volumes is comparable to the size of the viruses. Results of these studies can indirectly confirm an assumption of authors about possible transportation of viruses by erythrocytes to various organs and viral etiology of renal diseases with the hematuria syndrome and cervical cancer.

Keywords: graphene, graphene oxide, silver nanoparticles, structural and electrical properties, nephropathy, diagnostics.

\section{Introduction}

To increase the contrast of images in the study of biological objects by electron microscopy, the surfaces of the materials under investigation are deposited by metals ( $\mathrm{Au}, \mathrm{Pt}, \mathrm{Ni}$, for example), which usually makes it difficult to study nanoscale particles. Currently using a new class of electron microscopes to study the morphology of biological objects at low voltages and without metal deposition of the object [1-3]. It is known, that the effects of a dielectric on the surface of the slide significantly 
degrade image quality due to the accumulation of electric charge. In this regard, for the study of red blood cells we have suggested a method of forming a nanostructured substrate with the use of the reduced graphene oxide (GO). Due to the fact, that the $\mathrm{GO}$ substrates contain oxygen groups, the total electrical conductivity of the system increases by their removal and introduction of silver $(A g)$ nanoparticles. Earlier, we proved that with the help of electron microscopy it becomes possible to reveal changes in the morphology of erythrocytes in pathology and is likely we can investigate the presences of biological particles (viruses) on the surface of red cell [1-3].

The purpose of this study is development of methodology of formation of a nanostructured substrate of a composite of silver-graphene oxide for the study of changes in the morphology of erythrocytes by scanning electron microscopy in pathology.

\section{Materials and methods}

The GO suspension was obtained by graphite oxidation by a modified Hummers method [4-6]. To this end, a solution was prepared from $0.1 \mathrm{~g}$ of graphite powder, $0.05 \mathrm{~g}$ of sodium nitrate and concentrated sulfuric acid of $7 \mathrm{ml}$ with the addition of 0.3 $\mathrm{g}$ of potassium permanganate. The solution was stirred for 4 weeks. After dilution with deionized water up to $25 \mathrm{ml}$, a solution of hydrogen peroxide ( $5 \%$ ) was added to the mixture. The resulting yellow-yellow slurry was filtered to form a gel-like mass. The solution was then diluted with $50 \mathrm{ml}$ of water and sonicated at $750 \mathrm{~W}$ for $5 \mathrm{~min}$. The not exfoliated graphite oxide sheets were removed by centrifugation at $14500 \mathrm{rpm}$ for $5 \mathrm{~min}$. As a result, a homogeneous suspension of graphene oxide was obtained. To prepare the suspension with silver nanoparticles, aqueous solutions of sodium citrate $(1 \mathrm{ml})$ and silver nitrate $(0.25 \mathrm{ml})$ were successively added to $1.25 \mathrm{ml}$ of water under constant stirring at room temperature. After 5 minutes of incubation and addition of boiling water, the color of the solution changes from colorless to cloudy brown. The resulting solution was additionally boiled for 1 hour with continuous stirring. Further, the solutions of silver nanoparticles and GO were mixed in volume ratio of 1:1. The prepared slurry was deposited on a Si substrate and dried at room temperature for 24 hours. Dielectric substrates were pre-washed in acetone and alcohol in an ultrasonic bath, to impart hydrophilic properties, were treated in an aqueous solution of hydrogen peroxide and ammonia. The resulting samples were partially reduced by treatment at a temperature of $523 \mathrm{~K}$ for 30 minutes in an argon atmosphere in order to reduce the content of oxygen functional groups in the $\mathrm{GO}$. 
Scanning electron microscopy (JEOL JSM 780oF) with the integrated attachment of the X-ray energy dispersive spectrometer (EDS) (Inca Energy), atomic force microscopy (AFM) (Ntegra Spectra) and Raman spectroscopy (Ntegra Spectra) are used to characterize the nanocomposite properties. The electron microscope is equipped with a super-hybrid objective lens and the "Gentle Beam" system, which allows to obtain high-resolution images at low accelerating voltages, which is important for the study of biological objects. The use of such systems makes it possible to obtain images of the erythrocyte surface without the deposition of electrically conductive coatings. Accelerating voltages of $6 \mathrm{kV}$ and $1 \mathrm{kV}$, respectively, were used to study silver nanoparticles and blood smears. The current-voltage characteristics were measured by the fourprobe Van der Pau method (Ecopia 5500 HMS).

\section{Results and discussion}

Electron microscopic images of the rGO-Ag nanocomposite surface with various magnifications are shown in Figure 1a-c. Planar sizes of nanoparticles are in the range from 20 to $200 \mathrm{~nm}$. More than $60 \%$ of the particles have sizes from 60 to $100 \mathrm{~nm}$. Measurements by the EDS method in the light areas in Figure 1a-c showed the presence of silver (22.1 at.\%). Additionally, oxygen (51.5 at.\%), silicon (9.0 at.\%) and carbon (17.4 at.\%) are contained. When measuring small areas using the EDS method, the scan size in which the elemental composition is determined may exceed the dimensions of the selected point. This leads to taking into account the content of elements ( $C, S i$ and 0$)$ in the attached areas. The high content of oxygen and silicon in the results obtained can be explained by the influence of the elemental composition of the $\mathrm{SiO}_{2}$ substrate.

To determine the thickness of rGO-Ag nanocomposite films, incisions were made by the tip of the metallic needle. The height of the edges of the formed step was measured by the AFM method. The edge cross section along the line, which shown in Figure $2 a$, is demonstrated in Figure $2 b$. Estimates of heights in different places showed that the thickness of the rGO-Ar nanocomposite films does not exceed $10 \mathrm{~nm}$. At the same time, both SEM and AFM measurements show that the lateral dimensions of nanoparticles are an order of magnitude larger.

The Raman spectra of rGO-Ag nanocomposite excited by laser radiation with a wavelength of $532 \mathrm{~nm}$ are shown in Figure 3. Bands $D$ and $G$ observed at $\sim 1350 \mathrm{~cm}^{-1}$ and $\sim 1600 \mathrm{~cm}^{-1}$, respectively, are the main modes of Raman observed in the GO. The band $\mathrm{G}$ corresponds to the optical phonons $\mathrm{E}_{2 g}$ in the center of the Brillouin zone. The band $D$ is associated with breathing modes of six-atom rings of carbon and requires 

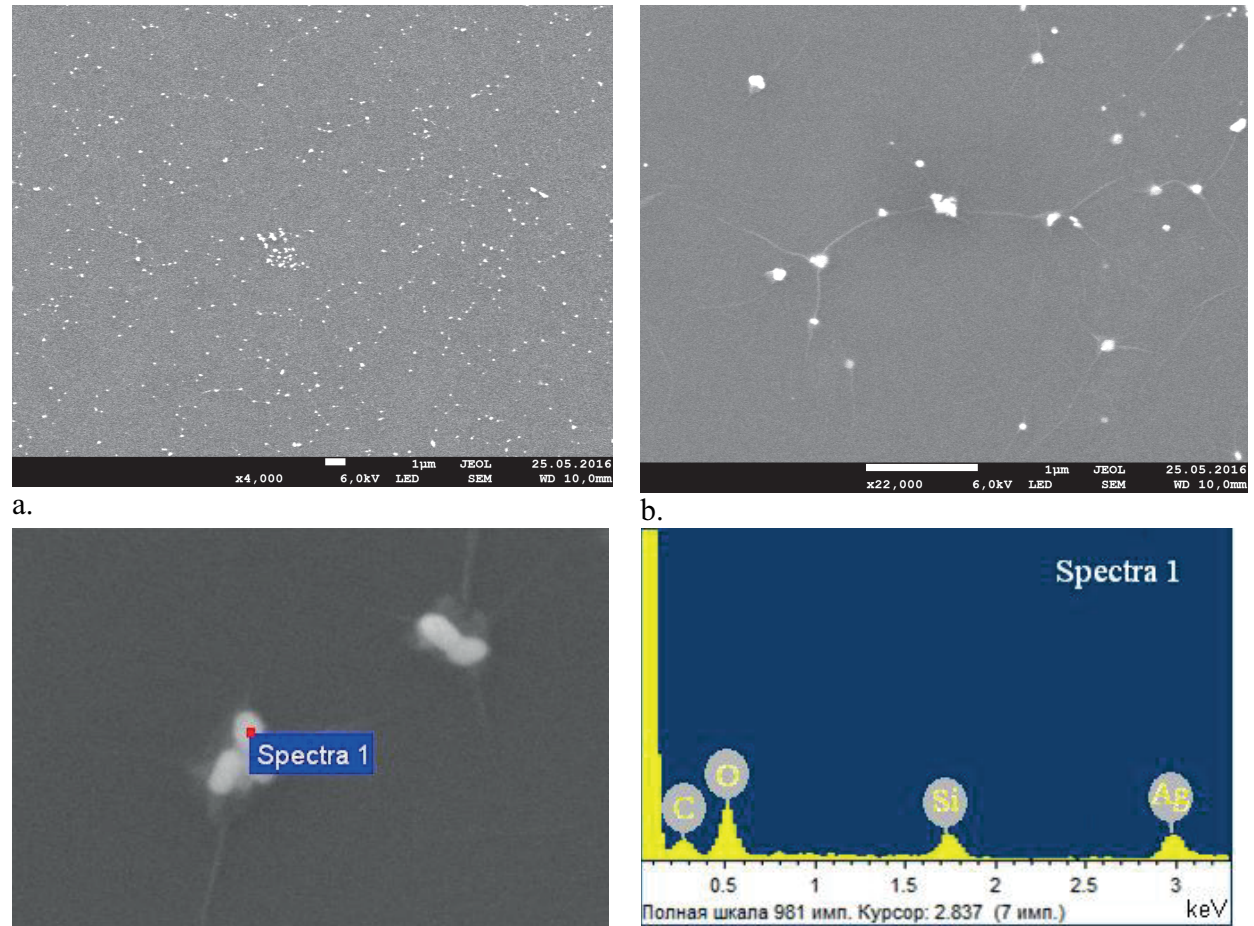

d.

Figure 1: SEM image of the surface of graphene oxide with silver nanoparticles at various magnifications $(a-c)$. EDS measurements at the point of Spectra 1 on Fig.1c (d).
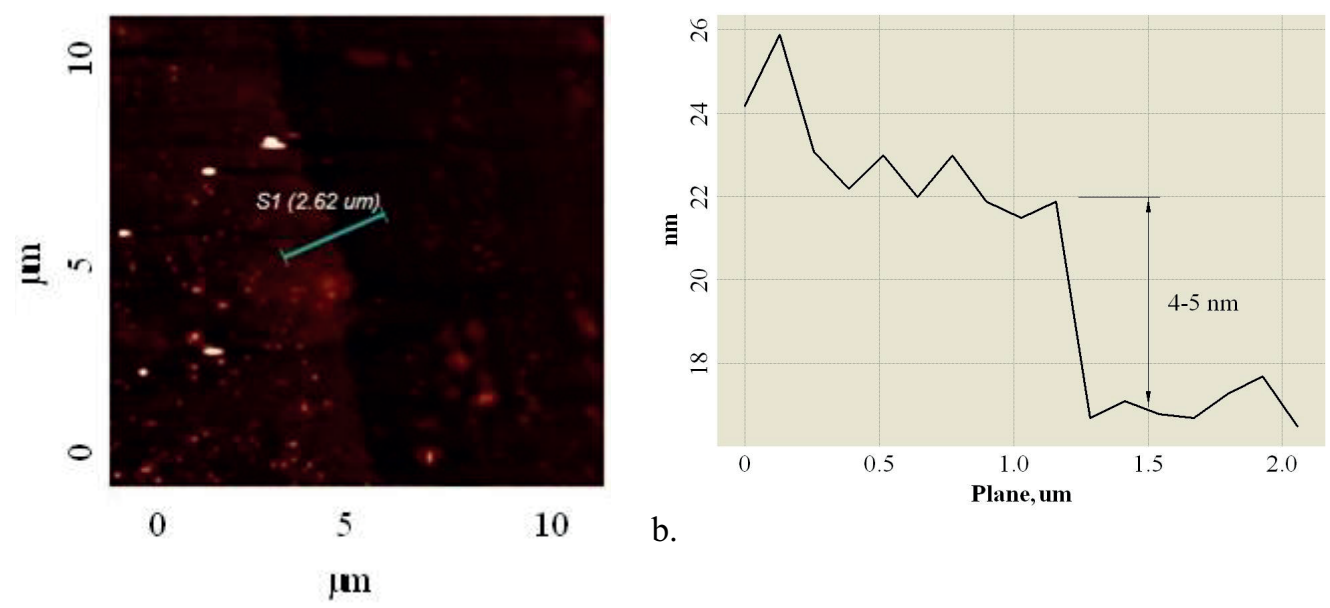

a.

Figure 2: AFM image of the threshold on the surface of the rGO-Ar nanocomposite (a) and its section (b).

the presence of defects for activation. The overtone $D$ peak ( $2 \mathrm{D}$ peak) is observed at $2700 \mathrm{~cm}^{-1}$ and is caused by a double resonance transition [7-10]. This peak, unlike the $D$ band, is observed even in the absence of defects. The band $D+G$ at $\sim 2930 \mathrm{~cm}^{-1}$ is also activated by defects. The ratio of the intensities of the D-and G-bands is widely used to characterize crystal disorder and defects in graphene-based materials [11, 12]. 
This ratio is 0.94 and 0.90 for the nanocomposite and GO respectively. It follows from this that when the rGO-Ag nanocomposite is formed, an insignificant increase in the defectiveness of the structure of the GO occurs.

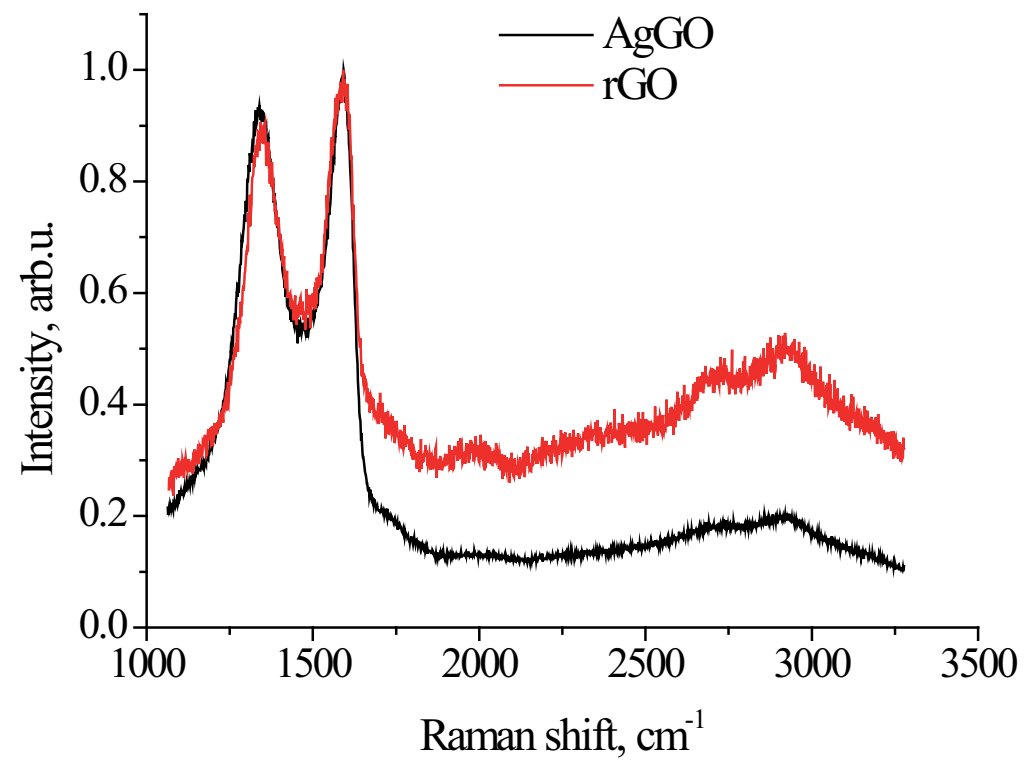

Figure 3: Raman spectra of rGO-Ag nanocomposite.

Figure 4 shows the temperature dependences of the sheet resistances of the rGO-Ag nanocomposite and reduced graphene oxide. The resistances were determined from the current-voltage characteristics, which had a linear form. As can be seen from the figure, over the entire temperature range from 80 to $450 \mathrm{~K}$, the values of the layer resistances of the rGO-Ag nanocomposite are 40 to 50 times less than for the $\mathrm{GO}$. The decrease in the resistance of GO upon introduction of silver nanoparticles can be due to both an increase in the concentration of charge carriers [13] and a modification of the mechanism of charge carrier transport $[14,15]$.

The obtained rGO-Ag composite was used to study the viral etiological factor of nephropathies with hematuria syndrome. The presence of such nanoobjects on the surface of erythrocytes was observed in patients with diseases of Berger and other types of nephropathy $[2,3]$, as well as for patients with cervical cancer. Figure 5 shows images of the surface of a red blood cell sample of a girl of 10 years with chronic glomerulonephritis at magnifications of $50,000 \times$ on a substrate of glass. Also on the glass substrate the image of the surface of a red blood sample of a patient 47 years old obtained which has diagnosis of cancer of the cervix at a magnification of 20,000x (Figure 6). Figure 7 demonstrate images of dysmorphic erythrocyte surfaces at magnifications of 10,000x and 20,000x a blood sample on the rGO-NPs nanocomposite substrate. Figure 8 and 9 are images of the surfaces of red blood cells with nanosize particles in the case of chronic glomerulonephritis on nanostructured 


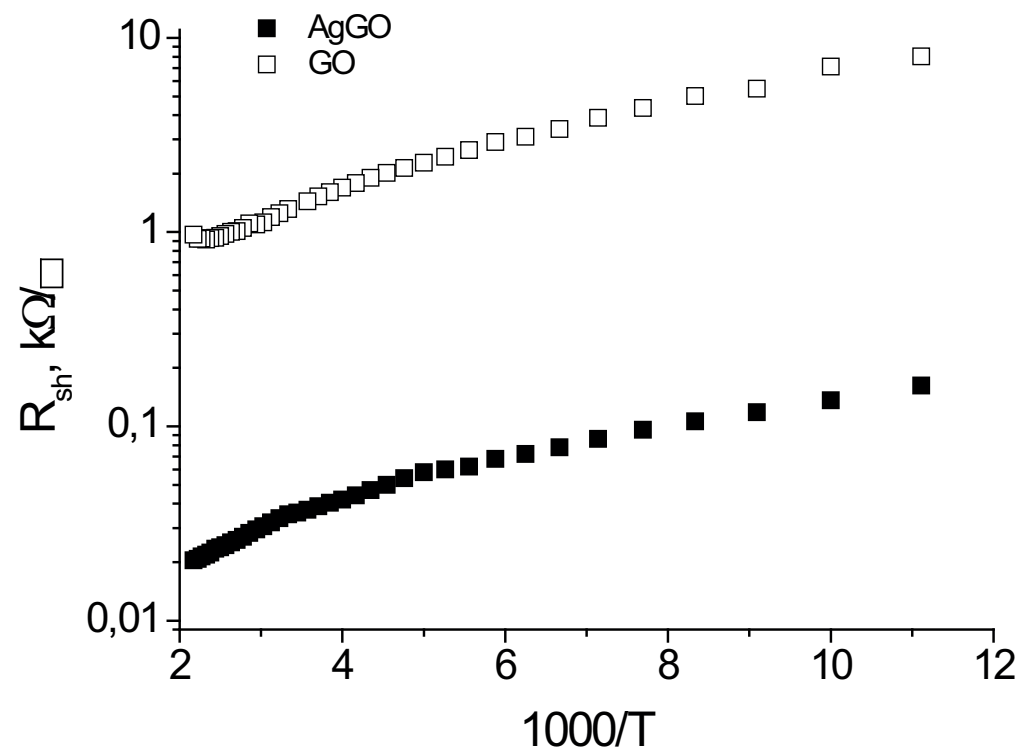

Figure 4: Sheet resistance of rGO and rGO_Ag nanocomposite as a function of 1000/T.

substrates at a magnification of 100,000x and in the case of cancer of the cervix at a magnification of 30,000x. Obtained images show that the nanoobjects on the surface of red blood cells differ in size and shape in various diseases. Studies have shown that SEM images of nanoscale objects on the surface of erythrocytes on a glass substrate start to become a blur (blurring) at magnifications of more than $50,000 x$, while on nanostructured substrates image of nanoparticles of good enough quality is obtained at a magnification of $100,000 \times$. In addition, at the increase $30,000 \times$ there is a difference of shapes of nanoobjects on the substrate surface of graphene oxide, while on glass substrates is not possible to observe.

Studies in the case of cervical cancer during radiotherapy show a change in the morphology of erythrocytes, the presence of virus-like nanoparticles and their number depending on the duration of therapy. In contrast to the images obtained on slides $[1,2]$, these images clearly show bright nanoscale biological objects, which can be attributed to viruses of similar sizes. The nature of these objects is not related to silver nanoparticles, since for their observation in an electron microscope an accelerating voltage of $\sim 6 \mathrm{kV}$ is required, while a voltage of $1 \mathrm{kV}$ is used here. In addition, elemental analysis carried out by the EDS method showed that these objects do not contain silver and contain carbon (77.8 at.\%), oxygen (19.1 at.\%), sodium (1.7 at.\%), sulfur (0.8 at.\%) and iron (0.6 at.\%). From the chemical composition of the object, it can be assumed that they are of an organic nature. The authors made an assumption about the possible viral etiologic factor of these diseases. At the same time, blood smears on glass substrates were used in the experiments and the researchers failed to obtain clear images of these formations. The use of substrates from the rGO-Ag nanocomposite made it 


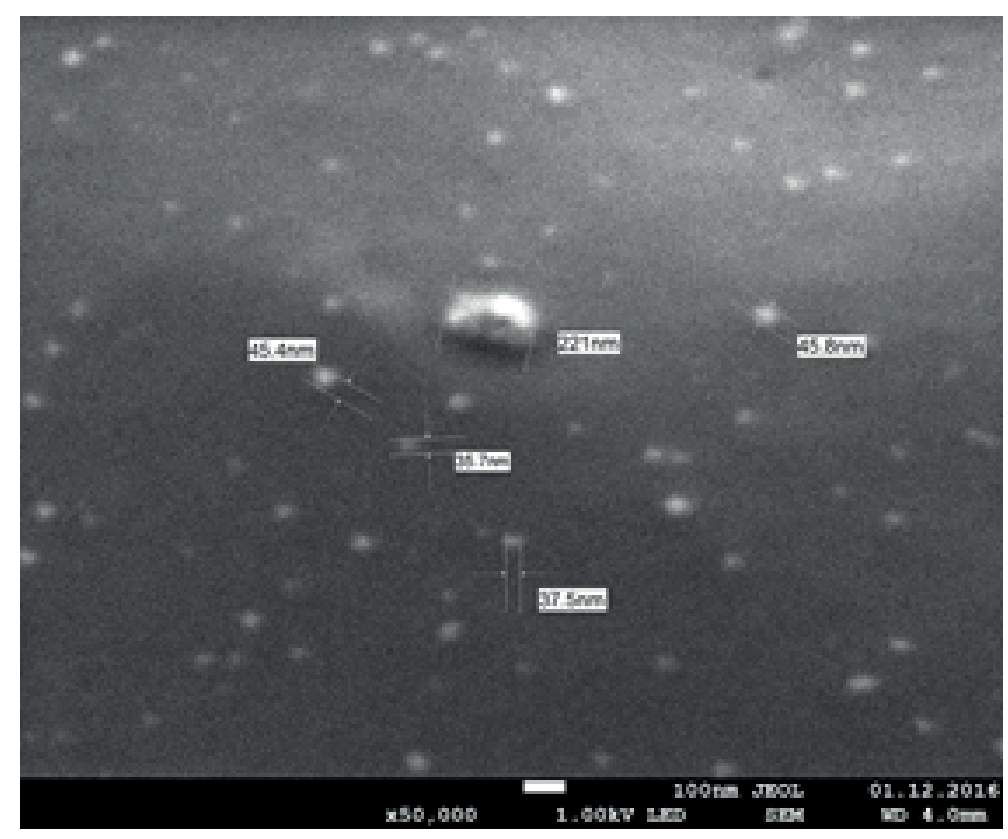

Figure 5: SEM image of nanometer particles with their sizes on the surface of erythrocytes on the dry clean polished glass in the case of glomerulonephritis, magnification 50,000x.

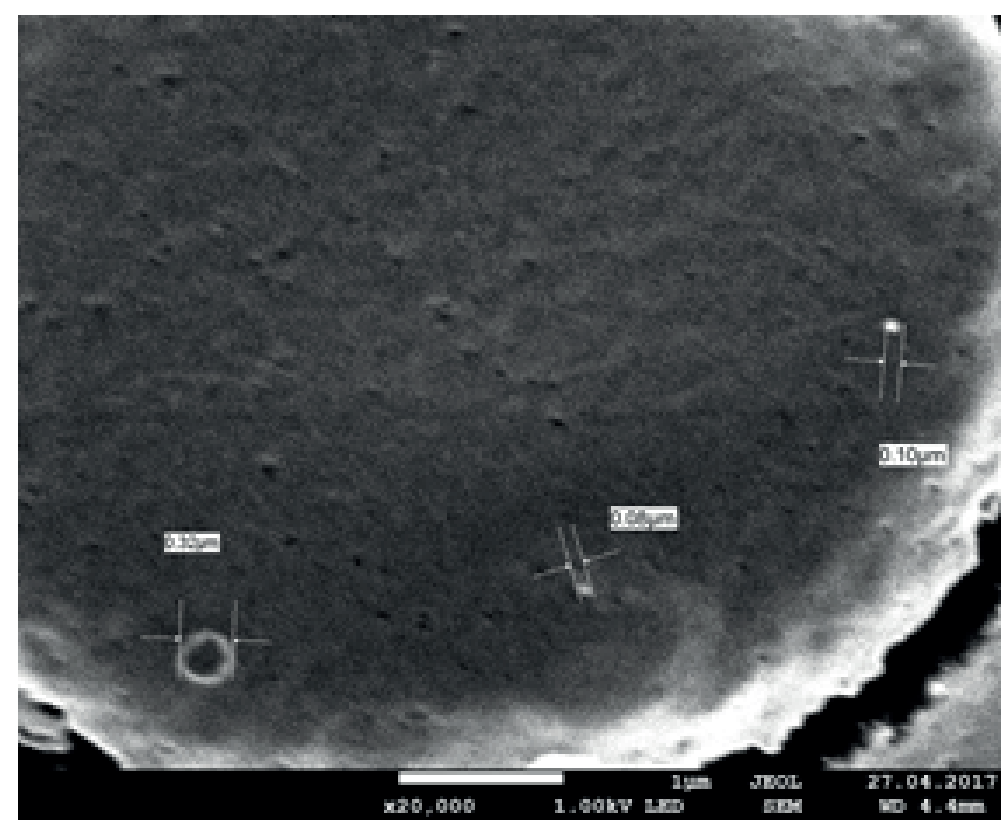

Figure 6: SEM image surface of erythrocyte of blood sample on the dry clean polished glass in case of cervical cancer, magnification 20,000x.

possible to more accurately reveal the shape and size of these objects (Figure 7-9). Improving the image quality of objects can be due to the elimination of accumulation of charges on the surface of dielectric materials (glass) under electron irradiation in SEM. Thus, the use of rGO-Ag nanocomposite substrates should improve the quality of diagnosing difficult types of nephropathy. 


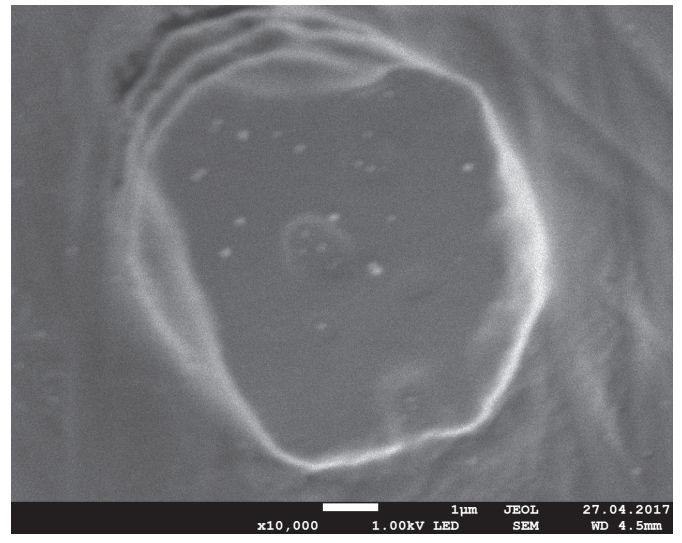

Figure 7: SEM images of nanoobjects on the surface of an dysmorphic erythrocyte on a rGO-Ag

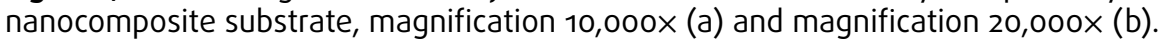

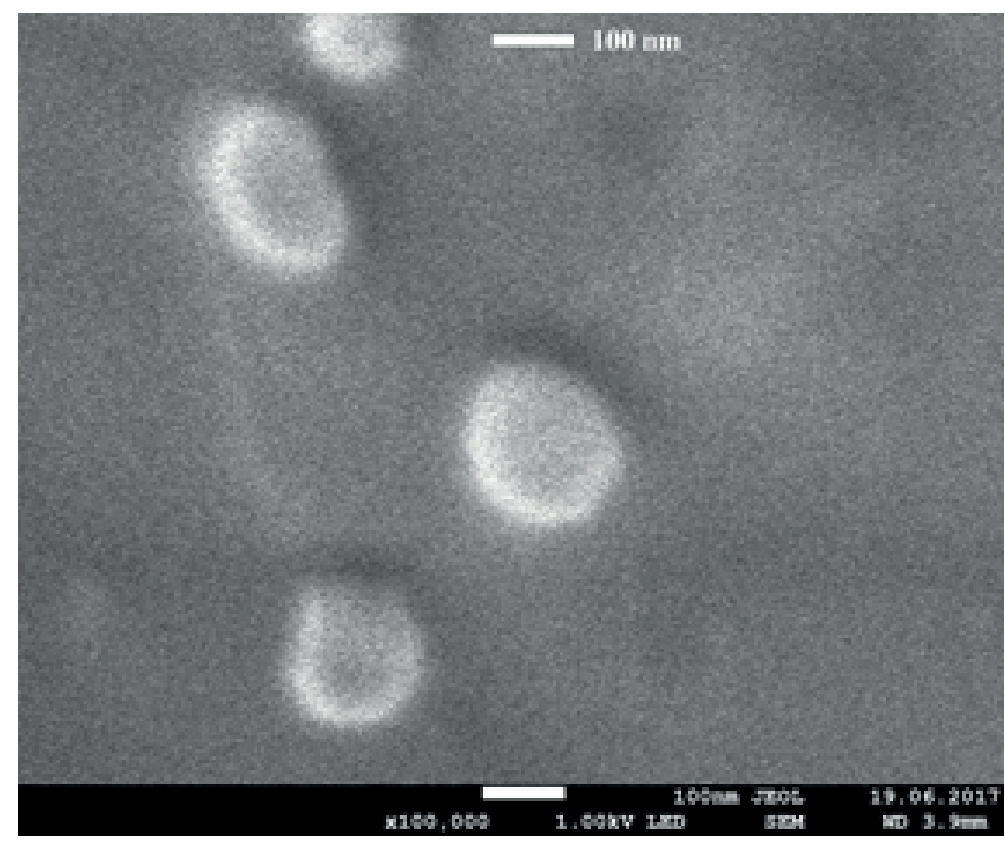

Figure 8: SEM image surface of erythrocyte of blood sample on substrate made from graphene oxide with silver nanoparticles in case of chronic glomerulonephritis, magnification 100,000x.

\section{Conclusion}

From the studies of electrical characteristics, it has been established that the synthesized rGO-Ag nanocomposite exhibits higher values of electrical conductivity than the pristine partially reduced samples of GO The use of substrates from this composite material allows more accurately reveal the shapes and sizes of biological nanoparticles. Improving the image quality of objects can also be due to the elimination of effects associated with the accumulation of dielectric materials (glass) on the surface under electron irradiation in SEM. Thus, nanocomposite rGO-Ag was used as a substrate for 


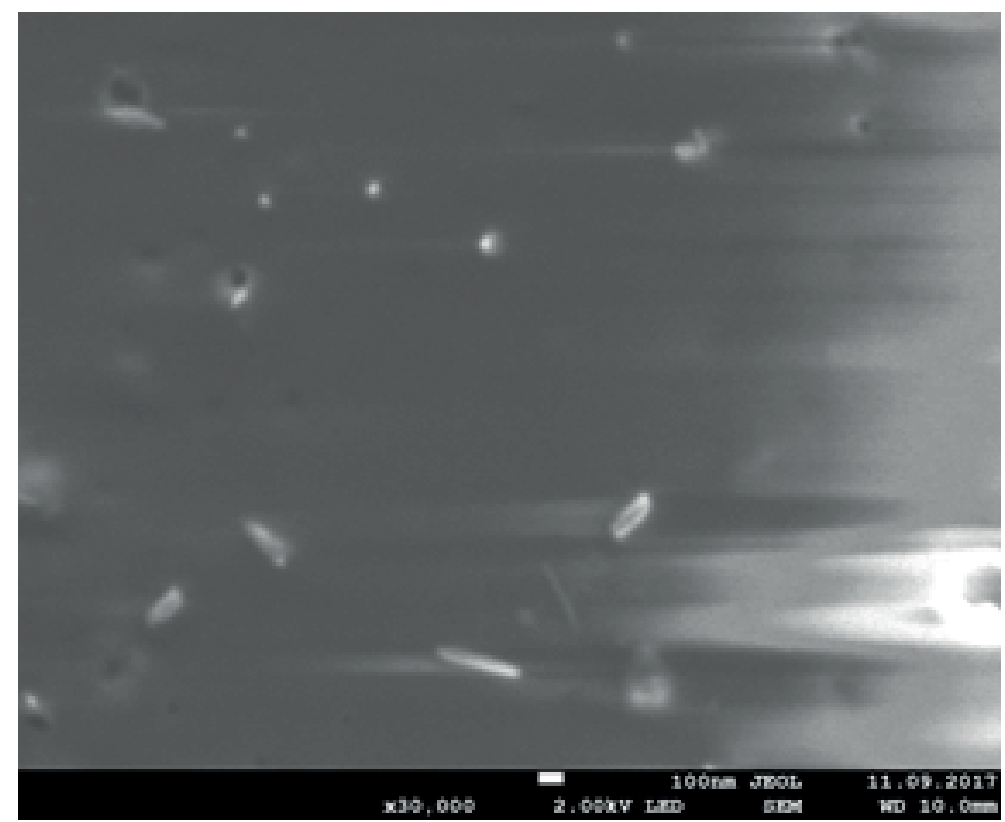

Figure 9: SEM image surface of erythrocyte of blood sample on substrate made from graphene oxide with silver nanoparticles in case of cervical cancer magnification 30,000x.

the study of blood smears, which were taken from patients with revealed signs of macrohematuria, by the SEM method. Nanosize particles on the surface of erythrocyte were observed, the sizes of which are comparable to the sizes of the viruses. The results of the work are of interest for the formation of a non-invasive differential complex method for the study of difficult to diagnose types of nephropathies, as well as a method for monitoring radiation therapy for cervical cancer.

\section{Acknowledgments}

The results were obtained within the framework of the state assignment of the Ministry of Education and Science of the Russian Federation "Organization of Scientific Research" No. 16.8272.2017/VU.

\section{References}

[1] G.V. Maksimov, S.N. Mamaeva, S.R. Antonov, Y.A. Munkhalova, I.V. Kononova, I.Yu. Sheikin. "Measuring Erythrocyte Morphology by Electron Microscopy to Diagnose Hematuria," Measurement Techniques, vol. 59, no3, pp. 327-330, 2016.

[2] S.N. Mamaeva, Y.A. Munkhalova, I.V. Kononova, A.A. Dyakonov, V.N. Koryakina, V.V. Shutova, G.V. Maksimov. "Issledovanie ehritrocitov krovi metodom rastrovoj ehlektronnoj mikroskopii," Russian Journal Vestnik Mordovskogo universiteta, vol. 
26, no 3. - pp. 381-390, 2016.

[3] G.V. Maksimov, S.N. Mamaeva, S.R. Antonov, Y.A. Munkhalova, I.V. Kononova, I.Yu. Sheikin. "Measuring erythrocyte morphology by electron microscopy to diagnose hematuriaю Metrology," Quarterly supplement to the journal Measuring Techniques, no. 1. pp.47-52, 2016.

[4] W. S. Hummers, R. E. Offeman. "Preparation of Graphitic Oxide", Journal of the American Chemical Society, vol. 80, no 6, pp. 1339, 1958.

[5] H. Yu, B. Zhang, C. Bulin, R. Li, R. Xing. "High-efficient Synthesis of Graphene Oxide Based on Improved Hummers Method," Sci Rep., vol. 6, pp. 36143 (1-7), 2016.

[6] S. N. Alam, N. Sharma, L. Kumar. "Synthesis of Graphene Oxide (GO) by Modified Hummers Method and Its Thermal Reduction to Obtain Reduced Graphene Oxide (rGO)*," Graphene, vol.6, pp.1-18, 2017.

[7] A.C. Ferrari, J.C. Meyer, V. Scardaci, C. Casiraghi, M. Lazzeri, F. Mauri, S. Piscanec, D. Jiang, K. S. Novoselov, S. Roth, A.K. Geim. "Raman Spectrum of Graphene and Graphene Layers," Phys. Rev. Letters, vol. 97, Pp.187401 (1-4), 2006.

[8] P. Klar, E. Lidorikis, A. Eckmann, I. A.Verzhbitskiy, A. C. Ferrari, C. Casiraghi "Raman scattering efficiency of graphene," Physical Review B, vol. 87, pp. 205435 (1-12), 2013.

[9] X. Dıez-Betriu, S. Alvarez-Garcıa, C. Botas, P. Alvarez, J. Sanchez-Marcos, C. Prieto, R. Menendez, A. R. de Andres. Raman spectroscopy for the study of reduction mechanisms and optimization of conductivity in graphene oxide thin films. J. Mater. Chem. C, no 1, pp. 6905-6912, 2013.

[10] K. N. Kudin, B. Ozbas, H. C. Schniepp, R.K. Prud'homme, I.A. Aksay, R. Car. "Raman Spectra of Graphite Oxide and Functionalized Graphene Sheets. Nano Letters," vol. 8, no. 1, pp. 36-41, 2008.

[11] L. G. Cancado, A. Jorio, E. H. Martins. Ferreira, Stavale F., C. A. Achete, R. B. Capaz,M. V. O. Moutinho, A. Lombardo, T. S. Kulmala, A. C. Ferrari. "Quantifying Defects in Graphene via Raman Spectroscopy at Different Excitation Energies" Nano Lett., vol. 11. - pp.3190-3196, 2011.

[12] A. Eckmann, A. Felten, A. Mishchenko, L. Britnell, R. Krupke, K. S. Novoselov, C. Casiraghi. "Probing the Nature of Defects in Graphene by Raman Spectroscopy,"Nano Lett., vol. 12, pp. 3925-3930, 2012.

[13] S. Khan, J. Ali, Harsh, M. Husain, M. Zulfequar. "Synthesis of reduced graphene oxide and enhancement of its electrical and optical properties by attaching Ag nanoparticles Physica E, vol. 81, pp. 320-325, 2016. 
[14] L. Li, Y. Guo, X. Zhang and Y. Song. "Inkjet-printed highly conductive transparent patterns with water based Ag-doped grapheme," J. Mater. Chem. A, vol. 2, pp.19095-19101, 2014.

[15] Y. Zhou, J.Yang, X.Cheng, N.Zhao, H.Sun, D.Li. "Transparent and conductive reduced grapheme oxide/silver nanoparticles multilayer film obtained by electrical selfassembly process with graphene oxide sheets and silver colloid," RSC Adv., vol. 3, pp. 3391-3398, 2013. 